\title{
COMBINED USE OF VIDEO LARYNGOSCOPY AND FIBEROPTIC FOR AIRWAY MANAGEMENT IN A PATIENT WITH FIXED CERVICAL SPINE
}

\author{
NUPUR MODA ${ }^{1 *}$, NIRAJ KUMAR ${ }^{2}$ \\ ${ }^{1}$ Department of Anesthesia, Critical Care and Pain Medicine, IMS and Sum Hospital, Bhubaneswar, Odisha, India. ${ }^{2}$ Department of \\ Neroanesthesiology and Critical Care, JPNATC, AIIMS, New Delhi, India. Email: drnups@yahoo.com
}

Received: 09 May 2018, Revised and Accepted: 29 June 2018

\begin{abstract}
Airway management may be difficult in patients with fixed cervical spine who have undergone previous spine surgery. Among the various techniques, fiber-optic intubation is a preferred method for securing the airway in such situation. However, it has some limitations also like identification of landmarks, especially in a case of distorted anatomy of the airway. To overcome this inadequacy, we used video laryngoscopy as a complement, to guide the tip of bronchoscope beneath the epiglottis into the trachea and thus achieving the goal. We present a case of difficult airway of fixed cervical spine with distorted anatomy in which combined use of fiberoptic and video laryngoscopy was performed to secure the airway. In our opinion, this technique can be utilized for other difficult airway case scenario also.
\end{abstract}

Keywords: Cervical spine, Difficult airway, Fiber-optic intubation, Video laryngoscopy.

(c) 2018 The Authors. Published by Innovare Academic Sciences Pvt Ltd. This is an open access article under the CC BY license (http://creativecommons. org/licenses/by/4. 0/) DOI: http://dx.doi.org/10.22159/ajpcr.2018.v11i11.27199

\section{INTRODUCTION}

Securing the airway is a great challenge in a condition like previous cervical spine surgery with a fixed spine. Fixation of the cervical spine makes airway management more difficult because there is a restriction of neck movement and mouth opening which make direct laryngoscopy often difficult or impossible [1,2]. In cases of previous surgeries, anatomy also misleads us, and hence, some different technique has to be adopted for securing a safe airway. Such patients may be associated with neurological damage due to the abnormal flexion and extension of the cervical spine besides of the fact that they are difficult to intubate. Awake fiberoptic-guided intubation should be commonly adopted as a method of choice in such cases. However, it is also not a full proof technique, especially in a condition in which anatomy of the airway is disturbed. Here, we present a case scenario in a patient of cerebrovertebral junction anomaly with implant failure in whom anatomy was distorted because of previous surgery; fiber-optic bronchoscope along with videolaryngoscope was used to secure the airway successfully.

\section{CASE REPORT}

A 23-year-old male was diagnosed atlantoaxial dislocation 2 years back after falling wherein an occipitocervical fixation was done (Fig. 1).

His symptoms improved but again after a trivial fall his symptoms reappeared and, this time, he has been rescheduled for surgery, i.e.,transoral odontoidectomy. On preanesthetic checkup, his mouth opening was hardly one finger, there was a severe restriction of neck flexion and extension, Mallampati Grade IV (Fig. 2).

Considering these features, it was obvious that endotracheal intubation with direct laryngoscopy would not be an easy task. Since it was an anticipated difficult airway so awake fiber-optic intubation was planned, and the patient was explained accordingly. Simultaneously, it was also decided to keep difficult airway cart along with surgical tracheostomy kit ready in case intubation fails. On patient arrival to the operating room, the intravenous cannula was placed and standard monitors were attached. Midazolam $1 \mathrm{mg}$ and fentanyl $50 \mu \mathrm{g}$ were given as a mild sedation. The upper airway was anesthetized with intraoral lidocaine gel, supplemented with transtracheal and superior laryngeal nerve block. $7 \mathrm{~mm}$ endotracheal tube (ETT) was premounted over the fiber- optic bronchoscope for awake oral intubation. Supplemental oxygen was administered through nasal prongs. Even after several attempts of oral fiber-optic bronchoscopy, we were unable to identify the glottis opening and any midline structures like epiglottis, and procedure was unsuccessful. The reason could be because of the distorted anatomy of the airway as a consequence of previous surgery. We then decided to take the help of viedeolaryngoscope and even with that also we could only identify tip of epiglottis, after applying all the maneuvers. By visibly identifying the tip of epiglottis, we tried to pass gum elastic bougie into the glottis opening just behind epiglottis, but again, it was not successful. Considering the fact that we could identify the tip of epiglottis (as a midline structure) with videolaryngoscope, fiberoptic was again tried for intubation along with it. While one person holding the viedeolaryngoscope and showing tip of epiglottis, fiber-optic bronchoscope was advanced just behind the epiglottis by another person (Fig. 4).

This time, we were able to put the tip of scope inside the glottis opening. After confirming the carina, the ETT already mounted on the scope was advanced into the trachea.

By adopting this unique technique, we managed to secure the airway successfully. Throughout the intubation process, oxygen saturation did not fall below $96 \%$. Confirming tube placement with capnography and the bilateral equal air entry on auscultation, general anesthesia was started with propofol and rocuronium.

After an uneventful intraoperative course, the patient was shifted to the intensive care unit with ETT in situ considering difficult intubation and expected post-operative airway edema. The patient was successfully extubated uneventfully on the next day, after ensuring adequacy of breathing.

\section{DISCUSSION}

Airway management in a patient with fixed cervical spine who has undergone previous surgery is very difficult and challenging. In our case, the main problem was the distorted anatomy of airway which made impossible to locate the midline structures. Awake fiber-optic intubation is regarded as a method of choice to secure the airway in case of anticipated difficult airway [3]. However, Johnson et al. found that with fiber-optic bronchoscopy-guided endotracheal intubation 


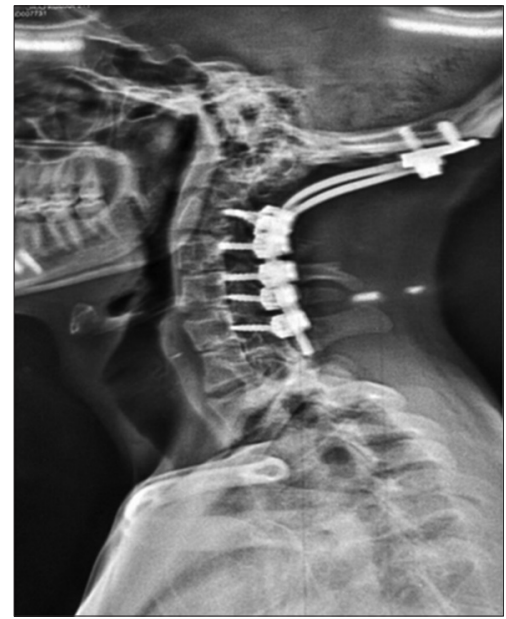

Fig. 1: Occipitocervical fixation

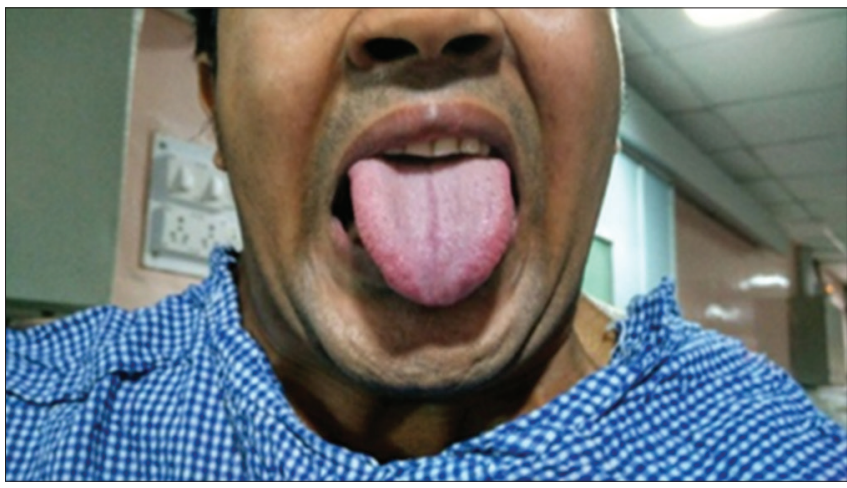

Fig. 2: Mallampati Grade IV and he had short neck and head slightly tilted to the left side (Fig. 3)

the failure rate of the first attempt is approximately 53.3\%, which denotes it demands great skills and experience [4]. Due to distorted anatomy and deviated neck to the left side fiberoptic alone were not successful, as we could not locate any structures. Even with the use of videolaryngoscope, we could not see more than the tip of epiglottis, so we used bougie but unsuccessful and avoided repeated attempt as doing so it would have been traumatic to the airway. Hence, it was decided to use fiberoptic so that we can visualize the structures as we go further instead of repeating blind bougie insertion. Hence, we thought of using videolaryngoscope to identify some structures; we could visualize tip of epiglottis with videolaryngoscope and then with its help, we inserted fiber-optic bronchoscope to intubate the trachea successfully.

Here are various options for intubating such patients such as fiberoptic intubation, retrograde intubation, and videolaryngoscope alone or with bougie. Each technique has its own advantages and limitations. Regarding retrograde intubation, in our case, landmarks were vague so it would not have been possible; moreover, this is an invasive technique.

Videolaryngoscopes are known for improving glottic view and make intubation easier, but still, studies have conflicting results regarding its effect on cervical spine motion. Furthermore, during intubation, blood and secretions may cause difficulties for video laryngoscopy. Various studies have suggested that cervical spine movement is least with fiber-optic intubation. Combining both the techniques, i.e.,video laryngoscopy and fiberoptic, we could avoid the limitation of individual technique and supplement each other to achieve the final goal. Viedeolaryngoscope helped in identifying midline structure (tip of epiglottis) which was not possible with fiberoptic alone. Moreover, this approach provided a fairly acceptable degree of success in securing the airway without applying any external pressure or other manipulation,

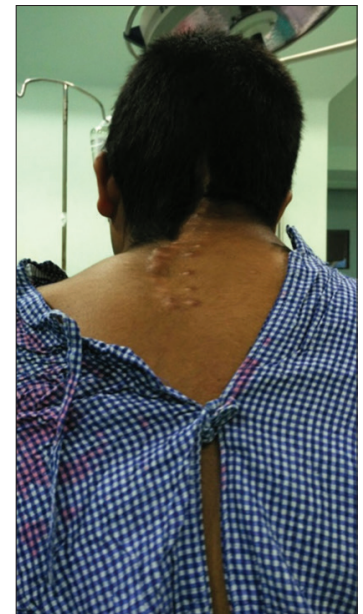

Fig. 3: Short neck and head slightly tilted to the left side

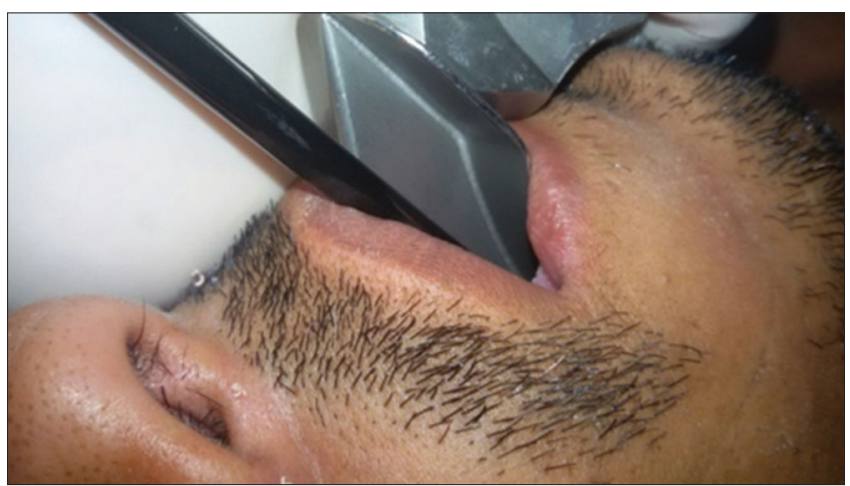

Fig. 4: Combined use of videolaryngoscope and fiber-optic bronchoscope

which was mandatory in our case. We used this technique as a tool of airway management, which is fixed cervical spine, but in future, it can be used for other difficult airway case scenario also. Gupta et al. have also shown a similar approach of video laryngoscopy added fiber-optic intubation for securing the airway in a patient with fixed spine [5]. Fiber-optic intubation needs experience, and it may cause airway obstruction and increases intracranial pressure. In an another case report, Uma et al. demonstrated nasal to oral conversion of the ETT with the help of Ryles tube in a patient of fixed cervical spine [6]. The urgency of the procedure and experience of the anesthetist are the main factors to decide which airway management should be used. However, there is not enough evidence to reveal the ideal intubation technique with least neurologic adverse effects.

\section{CONCLUSION}

Thus, fiber-optic bronchoscope along with videolaryngoscope is a great innovative option which can be considered in securing airway in cases of fixed cervical spine. This approach can be utilized in other cases of difficult airway management also. We suggest proper planning, preparation, and keep every option in account to deal with any circumstance in addition to case-specific strategies [7].

\section{ACKNOWLEDGMENT}

We want to thank the Department of Neuroanesthesia, CN Center, AIIMS, New Delhi, for giving us this opportunity.

\section{AUTHOR'S CONTRIBUTION}

First author performed the procedure. Second author guided through the procedure and writing of manuscript. Both authors discussed and contributed to the final manuscript. 


\section{CONFLICTS OF INTEREST}

There are no conflicts of interest.

\section{REFERENCES}

1. Schoenhage K, Koenig HM. Unanticipated difficult endotracheal intubations in patients with cervical spine instrumentation. Anesth Analg 2006;102:960-3.

2. Manninen PH, Jose GB, Lukitto K, Venkatraghavan L, El Beheiry H. Management of the airway in patients undergoing cervical spine surgery. J Neurosurg Anesthesiol 2007;19:190-4.

3. Jeffery LA, Carin AH, Robert AC. Practice guidelines for management of the difficult airway: An updated report by the American society of anesthesiologists task force on management of the difficult airway.
Anesthesiology 2013;118:1-20.

4. Johnson DM, From AM, Smith RB, From RP, Maktabi MA. Endoscopic study of mechanisms of failure of endotracheal tube advancement into the trachea during awake fiberoptic orotracheal intubation. Anesthesiology 2005;102:910-4.

5. Gupta N, Pandia MP, Prabhakar H, Chauhan M. Video laryngoscopy added fiberoptic intubation in a patient with difficult airway. J Anaesthesiol Clin Pharmacol 2013;29:283-4

6. Hariharan U, Ganguly N, Sood J, Shastri P. Airway management of fixed cervical spine undergoing transoral odontoidectomy: A novel technique. Curr Med Res Pract 2014;4:171-3.

7. Senapati LK, Samanta P. Effect of dexmedetomidine on dose requirement of propofol and thiopentone induction in patients under general endotracheal anesthesia. Asian J Pharm Clin Res 2018;11:262-5. 